\title{
Fatou's Lemma for Weakly Converging Measures under the Uniform Integrability Condition
}

\author{
Eugene A. Feinberd1, Pavlo O. Kasyanov, and Yan Liand
}

\begin{abstract}
This note describes Fatou's lemma for a sequence of measures converging weakly to a finite measure and for a sequence of functions whose negative parts are uniformly integrable with respect to these measures. The note also provides new formulations of uniform Fatou's lemma, uniform Lebesgue convergence theorem, the Dunford-Pettis theorem, and the fundamental theorem for Young measures based on the equivalence of uniform integrability and the apparently weaker property of asymptotic uniform integrability for sequences of functions and finite measures.
\end{abstract}

\section{Introduction}

Fatou's lemma states under appropriate conditions that the integral of the lower limit of a sequence of functions is not greater than the lower limit of the integrals. This inequality holds under one of the following conditions: (i) each function is nonnegative; (ii) the sequence of functions is bounded below by an integrable function; (iii) the sequence of negative parts of the functions is uniformly integrable; see Shiryaev [30, pp. 187, 211]. Serfozo [29, Lemma 3.2] established Fatou's lemma for a sequence of measures converging vaguely on a locally compact metric space and for nonnegative functions. Feinberg et al. [15, Theorem 4.2] provided Fatou's lemma for a sequence of measures converging weakly and for functions bounded below by a sequence of functions satisfying a certain minorant condition, which is satisfied for nonnegative functions. In this note we establish Fatou's lemma for a sequence of measures converging weakly and for functions whose negative parts satisfy the uniform integrability condition.

Uniform integrability of a family of functions plays an important role in probability theory and analysis. The relevant notion is asymptotic uniform integrability of a sequence of random variables [34, p. 17]. In this note we introduce the definitions of uniformly integrable (u.i.) and asymptotically uniformly integrable (a.u.i.) functions with respect to (w.r.t.) to a sequence of finite measures, and we show that these definitions are equivalent. This equivalence provides alternative formulations and proofs to some facts that use uniform integrability or asymptotic uniform integrability assumptions. For the case of a single probability measure, this equivalence is established in Kartashov [25, p. 180].

\footnotetext{
${ }^{1}$ Department of Applied Mathematics and Statistics, Stony Brook University, Stony Brook, NY 11794-3600, USA, eugene.feinberg@sunysb.edu

${ }^{2}$ Institute for Applied System Analysis, National Technical University of Ukraine "Igor Sikorsky Kyiv Polytechnic Institute", Peremogy ave., 37, build, 35, 03056, Kyiv, Ukraine, kasyanov@i.ua.

${ }^{3}$ Department of Applied Mathematics and Statistics, Stony Brook University, Stony Brook, NY 11794-3600, USA, yan.liang@stonybrook.edu
} 
Fatou's lemmas for weakly converging measures have significant applications to various areas and fields including stochastic processes [5, 7, 21, 26], statistics [19, 31, 32], control [6, 12, 14, 17, 35], game theory [22], functional analysis [20], optimization [37, and electrical engineering [28]. Our initial motivation in studying Fatou's lemma for variable probabilities was caused by its usefulness for the proof of the validity of optimality inequalities and the existence of stationary optimal policies for infinite-horizon, Borel-state, average-cost Markov decision process with noncompact action sets and unbounded costs [14]. These results have significant applications to inventory control [11, 18].

Other versions of Fatou's lemmas for variable measures are also important for applications. The recently discovered uniform Fatou lemma and uniform Lebesgue convergence theorems [16] play the central role in establishing sufficient optimality conditions for partially observable Markov decision processes with Borel state and action spaces [17. Unlike the classic Fatou's lemma, which provides sufficient conditions for Fatou's inequality, the uniform Fatou lemma states necessary and sufficient condition for the uniform version of Fatou's inequality. If all the functions are absolutely integrable, these necessary and sufficient conditions are more general than the conditions in the classic Fatou lemma. One of two necessary conditions in the uniform Fatou lemma [16, Theorem 2.1] is that the sequence of negative parts of functions is uniformly integrable with respect to the measures; see (2.1) below. The central result of this paper, Theorem 2.4, states that this condition is sufficient for the validity of Fatou's inequality for weakly converging measures. The examples in Section 3 show that this condition and the sufficient condition in Assumption 2.5, which was introduced in [15], do not imply each other. In particular, Theorem 2.4 is useful for studying Markov decision processes and stochastic games with cost functions unbounded from above and from below; see [9, 13, 23] where such problems are studied.

Fatou's lemma and Lebesgue's convergence theorems for probabilities are classic facts in probability theory. However, their versions for finite measures are also important for probability theory and its applications. This is the reason this paper and [16] study finite measures rather than probabilities. For example, the theory of optimization of Markov decision processes with multiple criteria is based on considering occupancy (also often called occupation) measures, which typically are not probability measures [4]. Another example is [26], where Fatou's lemma for nonnegative functions and finite measures is used.

Though uniform integrability and asymptotic uniform integrability properties of a sequence of functions with respect to a sequence of finite measures are equivalent, it is typically easier to verify asymptotic uniform integrability. This is important for applications. For this reason we provide in Section 4 alternative formulations of the uniform Fatou lemma and Lebesgue convergence theorem from [16] and two classic facts important for applications. In these formulations uniform integrability is substituted with asymptotic uniform integrability.

Section 2 of this paper provides definitions, describes the equivalence of uniform integrability and asymptotic uniform integrability, and states Fatou's lemma and Lebesgue's dominated convergence theorem for weakly converging measures. In particular, Fatou's lemma is formulated in Section 2 for weakly converging measures and for a.u.i. sequences of functions, which is equivalent to the assumption that the sequence of functions is u.i. Section 3 illustrates with the examples that the uniform integrability condition stated in Theorem 2.4 neither implies nor is implied by the minorant condition; see Assumption 2.5 and Corollary 2.7 below. Example 3.6 demonstrates that limsup in inequalities (2.7) in Assumption 2.5 cannot be relaxed to lim inf. By making use of the equivalence of uniform integrability and asymptotic uniform integrability, Section 4 provides alternative formulations of uniform Fatou's lemma, uniform Lebesgue's dominated convergence theorem, the Dunford-Pettis theorem, and Ball's fundamental theorem for Young measures. Section 5 provides the proofs of Fatou's lemma for weakly converging measures and relevant statements formulated in Section 2 , 


\section{$2 \quad$ Main results}

Let $(\mathbb{S}, \Sigma)$ be a measurable space, $\mathcal{M}(\mathbb{S})$ be the family of all finite measures on $(\mathbb{S}, \Sigma)$, and $\mathbf{P}(\mathbb{S})$ be the family of all probability measures on $(\mathbb{S}, \Sigma)$. When $\mathbb{S}$ is a topological space, as a rule, we consider $\Sigma:=\mathcal{B}(\mathbb{S})$, where $\mathcal{B}(\mathbb{S})$ is the Borel $\sigma$-field on $\mathbb{S}$. Let $\mathbf{R}$ be the real line and $\overline{\mathbf{R}}:=[-\infty,+\infty]$. The notation $\mathbf{I} A$ denotes the indicator of the event $A$.

Throughout this paper, we deal with integrals of functions that can take both positive and negative values. An integral $\int_{\mathbb{S}} f(s) \mu(d s)$ of a measurable $\overline{\mathbf{R}}$-valued function $f$ on $\mathbb{S}$ with respect to a measure $\mu$ is defined and equal to

$$
\int_{\mathbb{S}} f(s) \mu(d s)=\int_{\mathbb{S}} f^{+}(s) \mu(d s)-\int_{\mathbb{S}} f^{-}(s) \mu(d s),
$$

if

$$
\min \left\{\int_{\mathbb{S}} f^{+}(s) \mu(d s), \int_{\mathbb{S}} f^{-}(s) \mu(d s)\right\}<\infty,
$$

where $f^{+}(s)=\max \{f(s), 0\}, f^{-}(s)=-\min \{f(s), 0\}, s \in \mathbb{S}$. All the integrals in the assumptions of the theorems and corollaries throughout this paper are assumed to be defined.

Definition 2.1. The sequence of measurable $\overline{\mathbf{R}}$-valued functions $\left\{f_{n}\right\}_{n=1,2, \ldots}$ is called

- uniformly integrable (u.i.) w.r.t. a sequence of measures $\left\{\mu_{n}\right\}_{n=1,2, \ldots} \subset \mathcal{M}(\mathbb{S})$ if

$$
\lim _{K \rightarrow+\infty} \sup _{n=1,2, \ldots} \int_{\mathbb{S}}\left|f_{n}(s)\right| \mathbf{I}\left\{s \in \mathbb{S}:\left|f_{n}(s)\right| \geq K\right\} \mu_{n}(d s)=0
$$

- asymptotically uniformly integrable (a.u.i.) w.r.t. a sequence of measures $\left\{\mu_{n}\right\}_{n=1,2, \ldots} \subset$ $\mathcal{M}(\mathbb{S})$ if

$$
\lim _{K \rightarrow+\infty} \limsup _{n \rightarrow \infty} \int_{\mathbb{S}}\left|f_{n}(s)\right| \mathbf{I}\left\{s \in \mathbb{S}:\left|f_{n}(s)\right| \geq K\right\} \mu_{n}(d s)=0 .
$$

We remark that the limit as $K \rightarrow+\infty$ in (2.1) $((2.2))$ exists because the function

$$
K \mapsto \sup _{n=1,2, \ldots}\left(\limsup _{n \rightarrow \infty}\right) \int_{\mathbb{S}}\left|f_{n}(s)\right| \mathbf{I}\left\{s \in \mathbb{S}:\left|f_{n}(s)\right| \geq K\right\} \mu_{n}(d s)
$$

is nonincreasing in $K>0$.

If $\mu_{n}=\mu \in \mathcal{M}(\mathbb{S})$ for each $n=1,2, \ldots$, then an (a.)u.i. w.r.t. $\left\{\mu_{n}\right\}_{n=1,2, \ldots}$ sequence $\left\{f_{n}\right\}_{n=1,2, \ldots}$ is called $(a$.$) u.i. For a single finite measure \mu$, the definition of an a.u.i. sequence of functions (random variables in the case of a probability measure $\mu$ ) coincides with the corresponding definition broadly used in the literature; see, e.g., [34, p. 17]. Also, for a single fixed finite measure, the definition of a u.i. sequence of functions is consistent with the classic definition of a family $\mathcal{H}$ of u.i. functions. We say that a function $f$ is (a.)u.i. w.r.t. $\left\{\mu_{n}\right\}_{n=1,2, \ldots}$ if the sequence $\{f, f, \ldots\}$ is (a.)u.i. w.r.t. $\left\{\mu_{n}\right\}_{n=1,2, \ldots}$. A function $f$ is u.i. w.r.t. a family $\mathcal{N}$ of measures if

$$
\lim _{K \rightarrow+\infty} \sup _{\mu \in \mathcal{N}} \int_{\mathbb{S}}|f(s)| \mathbf{I}\{s \in \mathbb{S}:|f(s)| \geq K\} \mu(d s)=0 .
$$

The following theorem states the equivalence of the uniform and asymptotic uniform integrability properties introduced in Definition 2.1. The proof of Theorem 2.2 is presented in the Appendix. Several examples of applications of Theorem 2.2 are provided in Section 4 . As mentioned in the Introduction, for $\mu_{n}=\mu$ with $\mu$ being a probability measure, $n=1,2, \ldots$, Theorem 2.2 is presented in Kartashov [25, p. 180]. 
Theorem 2.2 (cp. Kartashov [25, p. 180]). Let $(\mathbb{S}, \Sigma)$ be a measurable space, $\left\{\mu_{n}\right\}_{n=1,2, \ldots}$ be a sequence of measures from $\mathcal{M}(\mathbb{S})$, and $\left\{f_{n}\right\}_{n=1,2, \ldots}$ be a sequence of measurable $\overline{\mathbf{R}}$-valued functions on $\mathbb{S}$. Then there exists $N=0,1, \ldots$ such that $\left\{f_{n+N}\right\}_{n=1,2, \ldots}$ is u.i. w.r.t. $\left\{\mu_{n+N}\right\}_{n=1,2, \ldots}$ if and only if $\left\{f_{n}\right\}_{n=1,2, \ldots}$ is a.u.i. w.r.t. $\left\{\mu_{n}\right\}_{n=1,2, \ldots}$.

We recall that Fatou's lemma claims that for a sequence of nonnegative measurable functions $\left\{f_{n}\right\}_{n=1,2, \ldots}$ defined on a measurable space $(\mathbb{S}, \Sigma)$ and for a measure $\mu$ on this space

$$
\int_{\mathbb{S}} \liminf _{n \rightarrow \infty} f_{n}(s) \mu(d s) \leq \liminf _{n \rightarrow \infty} \int_{\mathbb{S}} f_{n}(s) \mu(d s) .
$$

Although a sequence of functions is u.i. if and only if it is a.u.i., in many cases it is easier to verify that the sequence of functions is a.u.i. than that it is u.i.

Definition 2.3. A sequence of measures $\left\{\mu_{n}\right\}_{n=1,2, \ldots}$ on a metric space $\mathbb{S}$ converges weakly to a finite measure $\mu$ on $\mathbb{S}$ if for each bounded continuous function $f$ on $\mathbb{S}$

$$
\lim _{n \rightarrow \infty} \int_{\mathbb{S}} f(s) \mu_{n}(d s)=\int_{\mathbb{S}} f(s) \mu(d s) .
$$

The following theorem is the main result of this section. We provide the proof of this theorem in Section 5 .

Theorem 2.4 (Fatou's lemma for weakly converging measures). Let $\mathbb{S}$ be a metric space, $\left\{\mu_{n}\right\}_{n=1,2, \ldots}$ be a sequence of measures on $\mathbb{S}$ converging weakly to $\mu \in \mathcal{M}(\mathbb{S})$, and $\left\{f_{n}\right\}_{n=1,2, \ldots}$ be a sequence of measurable $\overline{\mathbf{R}}$-valued functions on $\mathbb{S}$ such that $\left\{f_{n}^{-}\right\}_{n=1,2, \ldots}$ is a.u.i. w.r.t. $\left\{\mu_{n}\right\}_{n=1,2, \ldots}$. Then

$$
\int_{\mathbb{S}} \liminf _{n \rightarrow \infty, s^{\prime} \rightarrow s} f_{n}\left(s^{\prime}\right) \mu(d s) \leq \liminf _{n \rightarrow \infty} \int_{\mathbb{S}} f_{n}(s) \mu_{n}(d s)
$$

Consider the following assumption introduced in [15], which is a sufficient condition for the validity of Fatou's lemma for weakly converging measures.

Assumption 2.5. Let $\mathbb{S}$ be a metric space, $\left\{\mu_{n}\right\}_{n=1,2, \ldots}$ be a sequence of measures on $\mathbb{S}$ that converges weakly to $\mu \in \mathcal{M}(\mathbb{S})$, and $\left\{f_{n}, g_{n}\right\}_{n=1,2, \ldots}$ be a sequence of measurable $\overline{\mathbf{R}}$-valued functions on $\mathbb{S}$ such that $f_{n}(s) \geq g_{n}(s)$ for each $n=1,2, \ldots$ and $s \in \mathbb{S}$, and

$$
-\infty<\int_{\mathbb{S} n \rightarrow \infty, s^{\prime} \rightarrow s} \limsup _{n} g_{n}\left(s^{\prime}\right) \mu(d s) \leq \liminf _{n \rightarrow \infty} \int_{\mathbb{S}} g_{n}(s) \mu_{n}(d s) .
$$

We note that Assumption 2.5 implies under certain conditions that the sequence of functions $\left\{f_{n}^{-}\right\}_{n=1,2, \ldots}$ is u.i. w.r.t. $\left\{\mu_{n}\right\}_{n=1,2, \ldots}$; see Theorem 2.6 below. In general, these two conditions do not imply each other; see Examples 3.1 and 3.2. The following theorem, whose proof is provided in Section 5, describes a sufficient condition when uniform integrability is more general than Assumption 2.5. In addition, according to Example 3.1, these two assumptions are not equivalent under the sufficient condition stated in Theorem 2.6.

Theorem 2.6. Let Assumption 2.5 hold. If the sequence of functions $\left\{g_{n}\right\}_{n=1,2, \ldots}$ is uniformly bounded from above, then there exists $N=0,1, \ldots$ such that $\left\{f_{n+N}^{-}\right\}_{n=1,2, \ldots}$ is u.i. w.r.t. $\left\{\mu_{n+N}\right\}_{n=1,2, \ldots}$.

For weakly converging probability measures, Fatou's lemma is introduced in [29] and is generalized in [15, Theorem 4.2]. The following corollary extends [15, Theorem 4.2] to finite measures. The proof of Corollary 2.7 is provided in Section [5. Example 3.3 demonstrates that Corollary 2.7 is incorrect if Assumption 2.5) is weakened by replacing lim sup with lim inf in formula (2.7). 
Corollary 2.7 (Fatou's lemma for weakly converging measures; cp. [15, Theorem 4.2]). Inequality (2.6) holds under Assumption 2.5.

The following corollary states Lebesgue's dominated convergence theorem for weakly converging measures. The similar statement is provided in Serfozo [29, Theorem 3.5] in the form of the necessary and sufficient condition for nonnegative functions and for locally compact spaces. Though local compactness is not used in the proof of [29, Theorem 3.5], there is a difference beween the cases of nonnegative and general functions. If the functions can take both positive and negative values, the converse statement to Corollary 2.8 does not hold. This can be seen from Example 3.2.

Corollary 2.8 (Lebesgue's dominated convergence theorem for weakly converging measures; cp. 29, Theorem 3.5]). Let $\mathbb{S}$ be a metric space, $\left\{\mu_{n}\right\}_{n=1,2, \ldots}$ be a sequence of measures on $\mathbb{S}$ that converges weakly to $\mu \in \mathcal{M}(\mathbb{S})$, and $\left\{f_{n}\right\}_{n=1,2, \ldots}$ be a sequence of measurable $\overline{\mathbf{R}}$-valued functions on $\mathbb{S}$ such that $\lim _{n \rightarrow \infty, s^{\prime} \rightarrow s} f_{n}\left(s^{\prime}\right)$ exists for $\mu$-a.e. $s \in \mathbb{S}$. If $\left\{f_{n}\right\}_{n=1,2, \ldots}$ is a.u.i. w.r.t. $\left\{\mu_{n}\right\}_{n=1,2, \ldots}$, then

$$
\lim _{n \rightarrow \infty} \int_{\mathbb{S}} f_{n}(s) \mu_{n}(d s)=\int_{\mathbb{S}} \lim _{n \rightarrow \infty, s^{\prime} \rightarrow s} f_{n}\left(s^{\prime}\right) \mu(d s) .
$$

Proof. The corollary directly follows from Theorem 2.4. applied to the sequences $\left\{f_{n}\right\}_{n=1,2, \ldots}$ and $\left\{-f_{n}\right\}_{n=1,2, \ldots}$.

The following assumption provides a sufficient condition for a sequence of measurable functions $\left\{f_{n}\right\}_{n=1,2, \ldots}$ to be u.i. w.r.t. a sequence of finite measures $\left\{\mu_{n}\right\}_{n=1,2, \ldots}$.

Assumption 2.9. Let $\mathbb{S}$ be a metric space, $\left\{\mu_{n}\right\}_{n=1,2, \ldots}$ be a sequence of measures on $\mathbb{S}$ that converges weakly to $\mu \in \mathcal{M}(\mathbb{S})$, and $\left\{f_{n}, g_{n}\right\}_{n=1,2, \ldots}$ be a sequence of pairs of measurable $\overline{\mathbf{R}}$-valued functions on $\mathbb{S}$ such that $\left|f_{n}(s)\right| \leq g_{n}(s)$ for each $n=1,2, \ldots$ and $s \in \mathbb{S}$, and

$$
\limsup _{n \rightarrow \infty} \int_{\mathbb{S}} g_{n}(s) \mu_{n}(d s) \leq \int_{\mathbb{S}} \liminf _{n \rightarrow \infty, s^{\prime} \rightarrow s} g_{n}\left(s^{\prime}\right) \mu(d s)<+\infty .
$$

Corollary 2.10 (Lebesgue's dominated convergence theorem for weakly converging measures; cp. [29, Theorem 3.3]). If Assumption 2.9 holds and $\lim _{n \rightarrow \infty, s^{\prime} \rightarrow s} f_{n}\left(s^{\prime}\right)$ exists for $\mu$-a.e. $s \in \mathbb{S}$, then equality (2.8) holds.

Proof. According to Theorem 2.6, applied to $\mathrm{f}_{n}(s):=-\left|f_{n}(s)\right|$ and $\mathrm{g}_{n}(s):=-g_{n}(s), n=1,2, \ldots$, $s \in \mathbb{S}$, Assumption 2.9 implies that $\left\{f_{n}\right\}_{n=1,2, \ldots}$ is u.i. w.r.t. $\left\{\mu_{n}\right\}_{n=1,2, \ldots}$. In view of Theorem 2.2, the rest of the proof follows from Corollary 2.8.

\section{Counterexamples}

The following two examples illustrate that uniform integrability of $\left\{f_{n+N}^{-}\right\}_{n=1,2, \ldots}$ for some $N=$ $0,1, \ldots$ neither implies nor is implied by Assumption 2.5. In Example 3.1, $\left\{f_{n}^{-}\right\}_{n=1,2, \ldots}$ is u.i. w.r.t. $\left\{\mu_{n}\right\}_{n=1,2, \ldots}$, but Assumption 2.5 does not hold.

Example 3.1. Consider $\mathbb{S}:=[0,1]$ endowed with the standard Euclidean metric and consider the probability measures

$$
\mu_{n}(C):=\int_{C} n \mathbf{I}\left\{s \in\left[0, \frac{1}{n}\right]\right\} \nu(d s), \quad \mu(C):=\mathbf{I}\{0 \in C\}, \quad C \in \mathcal{B}(\mathbb{S}), \quad n=1,2, \ldots,
$$


where $\nu$ is the Lebesgue measure on $[0,1]$. Then $\mu_{n}$ converges weakly to $\mu$ as $n \rightarrow \infty$. Let $f_{n}: \mathbb{S} \mapsto \mathbf{R}$, $n=1,2, \ldots$, be

$$
f_{n}(s)= \begin{cases}-i, & \text { if } s \in\left[\frac{1}{n}\left(1-2^{-(i-1)}\right), \frac{1}{n}\left(1-2^{-i}\right)\right), \quad i=1,2, \ldots \\ 0, & \text { otherwise. }\end{cases}
$$

Then $\int_{\mathbb{S}} f_{n}(s) \mathbf{I}\left\{s \in \mathbb{S}: f_{n}(s) \leq-K\right\} \mu_{n}(d s)=\sum_{i=\lceil K\rceil}^{\infty} \frac{-i}{2^{i}}=-\frac{\lceil K\rceil+1}{2^{\lceil K\rceil-1}}$ for each $K>0$ and for all $n=1,2, \ldots$. Since $\frac{[K\rceil+1}{2^{\mid K\rceil-1}} \rightarrow 0$ as $K \rightarrow+\infty$, the sequence $\left\{f_{n}^{-}\right\}_{n=1,2, \ldots}$ is u.i. w.r.t. $\left\{\mu_{n}\right\}_{n=1,2, \ldots}$. Now, we show that Assumption 2.5 does not hold. Consider an arbitrary sequence of measurable functions $\left\{g_{n}\right\}_{n=1,2, \ldots}$ such that $g_{n}(s) \leq f_{n}(s)$ for all $n=1,2, \ldots$ and for all $s \in \mathbb{S}$. Let us prove that (2.7) does not hold. Assume, on the contrary, that (2.7) holds. Let $G:=\limsup _{n \rightarrow \infty, s^{\prime} \rightarrow 0} g_{n}\left(s^{\prime}\right)$. Since $g_{n}(s) \leq f_{n}(s) \leq 0$, then $G \leq 0$. In view of (3.1), inequalities (2.7) become

$$
-\infty<G \leq \liminf _{n \rightarrow \infty} \int_{\mathbb{S}} g_{n}(s) \mu_{n}(d s) .
$$

Note that, if (2.7) is true for $\left\{g_{n}\right\}_{n=1,2, \ldots}$, then it is true for $\left\{\tilde{g}_{n}\right\}$ such that $\tilde{g}_{n}(s):=g_{n}(s)-C$, where $C \geq 0$. Therefore, we can select $\left\{g_{n}\right\}_{n=1,2, \ldots}$ such that $G \in\{-2,-3, \ldots\}$. Then we show that $\liminf _{n \rightarrow \infty} \int_{\mathbb{S}} g_{n}(s) \mu_{n}(d s)<G$. Observe that the definition of $G$ implies

$$
\lim _{m \rightarrow \infty} \sup _{n \geq m, s \in\left[0, \frac{1}{m}\right]} g_{n}(s) \leq G
$$

in fact, the equality takes place, but we do not need it. Then for every $\varepsilon>0$ there exists $N(\varepsilon)>0$ such that $g_{n}(s) \leq G+\varepsilon$ for all $n \geq N(\varepsilon)$ and for all $s \in\left[0, \frac{1}{N(\varepsilon)}\right]$. Therefore, $g_{n}(s) \leq \min \left\{G+\varepsilon, f_{n}(s)\right\}$ for all $s \in\left[0, \frac{1}{N(\varepsilon)}\right]$ and for all $n \geq N(\varepsilon)$, which implies

$$
\int_{\mathbb{S}} g_{n}(s) \mu_{n}(d s) \leq \int_{\mathbb{S}} \min \left\{G+\varepsilon, f_{n}(s)\right\} \mu_{n}(d s), \quad n \geq N(\varepsilon) .
$$

Let $\varepsilon \in(0,1)$. For $n \geq N(\varepsilon)$

$$
\begin{aligned}
& \int_{\mathbb{S}} \min \left\{G+\varepsilon, f_{n}(s)\right\} \mu_{n}(d s)=\int_{0}^{\frac{1}{n}\left(1-2^{G+1}\right)}(G+\varepsilon) \mu_{n}(d s)+\int_{\frac{1}{n}\left(1-2^{G+1}\right)}^{\frac{1}{n}} f_{n}(s) \mu_{n}(d s) \\
= & (G+\varepsilon)\left(1-2^{G+1}\right)+\sum_{i=-G}^{\infty} \frac{-i}{2^{i}}=(G+\varepsilon)\left(1-2^{G+1}\right)-\frac{-G+1}{2^{-G-1}}=G+\varepsilon-(1+\varepsilon) 2^{G+1},
\end{aligned}
$$

where, as follows from (3.2), the first equality holds because $f_{n}(s) \geq G+1>G+\varepsilon$ for $s \in$ $\left[0, \frac{1}{n}\left(1-2^{G+1}\right)\right)$ and $f_{n}(s) \leq G<G+\varepsilon$ for $s \in\left[\frac{1}{n}\left(1-2^{G+1}\right), \frac{1}{n}\right)$. As follows from (3.4) and (3.5), for every $\varepsilon \in(0,1)$,

$$
\liminf _{n \rightarrow \infty} \int_{\mathbb{S}} g_{n}(s) \mu_{n}(d s)<G+\varepsilon-2^{G+1},
$$

and, therefore, $\liminf _{n \rightarrow \infty} \int_{\mathbb{S}} g_{n}(s) \mu_{n}(d s) \leq G-2^{G+1}<G$, which contradicts the second inequality in (3.3). Hence, Assumption 2.5 does not hold.

In addition, Kamihigashi's [24, Example 5.1] of a sequence of functions, which is not u.i., demonstrates that Assumption 2.5] does not imply that $\left\{f_{n+N}^{-}\right\}_{n=1,2, \ldots}$ is u.i. w.r.t. $\left\{\mu_{n}\right\}_{n=1,2, \ldots}$ for some $N=0,1, \ldots$. The following example is a slight modification of [24, Example 5.1]. 
Example 3.2 (cp. Kamihigashi [24, Example 5.1]). Consider $\mathbb{S}:=[-1,1]$ endowed with the standard Euclidean metric. Let $\mu_{n}=\mu, n=1,2, \ldots$, be the Lebesgue measure on $\mathbb{S}$ and for $n=1,2, \ldots, s \in \mathbb{S}$

$$
f_{n}(s)= \begin{cases}-n, & \text { if } s \in\left[-\frac{1}{n}, 0\right) \\ n, & \text { if } s \in\left(0, \frac{1}{n}\right] \\ 0, & \text { otherwise }\end{cases}
$$

Then $\liminf \operatorname{in}_{n \rightarrow \infty} \int_{\mathbb{S}} f_{n}(s) \mathbf{I}\left\{s \in \mathbb{S}: f_{n}(s) \leq-K\right\} \mu(d s)=-1$ for each $K>0$, which implies that $\left\{f_{n}^{-}\right\}_{n=1,2, \ldots}$ is not a.u.i. Hence $\left\{f_{n+N}^{-}\right\}_{n=1,2, \ldots}$ is not u.i. for each $N=0,1, \ldots$; see Theorem 2.2 . For each $n=1,2, \ldots$, since $\int_{\mathbb{S}} \lim \sup _{n \rightarrow \infty, s^{\prime} \rightarrow s} f_{n}\left(s^{\prime}\right) \mu(d s)=\int_{\mathbb{S}} f_{n}(s) \mu(d s)=0$, we have that (2.7) holds for $\mu_{n}=\mu$ with $g_{n}=f_{n}$.

The following example demonstrates that Corollary 2.7 fails if inequalities (2.7) are replaced in Assumption 2.5 with

$$
-\infty<\int_{\mathbb{S}} \liminf _{n \rightarrow \infty, s^{\prime} \rightarrow s} g_{n}\left(s^{\prime}\right) \mu(d s) \leq \liminf _{n \rightarrow \infty} \int_{\mathbb{S}} g_{n}(s) \mu_{n}(d s) .
$$

Example 3.3. (Inequalities (3.6) hold, but inequality (2.6) and the second inequality in (2.7) do not hold).

Let $\mathbb{S}:=[0,+\infty), \mu_{n}(S)=\mu(S):=\int_{S} 2^{-s} d s, S \in \mathcal{B}(\mathbb{S})$,

$$
f_{n}(s):=-2^{n} \mathbf{I}\{s \in[n, n+1)\}, \quad \text { and } \quad g_{n}(s):=f_{n}(s)-\frac{2^{s-1}}{\ln 2} \sum_{k=0}^{2^{n}-1} \mathbf{I}\left\{s \in\left[\frac{2 k}{2^{n}}, \frac{2 k+1}{2^{n}}\right)\right\}
$$

for all $s \in[0,+\infty)$ and $n=1,2, \ldots$ Note that $f_{n}(s) \geq g_{n}(s)$ for all $s \in[0,+\infty)$ and $n=1,2, \ldots$ Also,

$$
\liminf _{n \rightarrow \infty, s^{\prime} \rightarrow s} f_{n}\left(s^{\prime}\right)=\limsup _{n \rightarrow \infty, s^{\prime} \rightarrow s} g_{n}\left(s^{\prime}\right)=0, \quad s \in[0,+\infty) .
$$

Indeed, $\lim _{n \rightarrow \infty, s^{\prime} \rightarrow s} f_{n}\left(s^{\prime}\right) \equiv 0$ because $f_{n}\left(s^{\prime}\right)=0$ for $s^{\prime} \in[0, s+1)$, when $n \geq\lfloor s\rfloor+2$ and $s \in[0,+\infty)$, where $\lfloor a\rfloor$ is the integer part of the real number $a \in \mathbf{R}$. Since $g_{n}(s) \leq 0$ for all $s \in[0,+\infty)$ and $n=1,2, \ldots$, the second equality in (3.7) follows from

$$
0 \geq \limsup _{n \rightarrow \infty, s^{\prime} \rightarrow s} g_{n}\left(s^{\prime}\right) \geq \lim _{n \rightarrow \infty} g_{n}\left(\frac{2\left\lfloor 2^{n-1} s\right\rfloor+\frac{3}{2}}{2^{n}}\right)=0, \quad s \in[0,+\infty),
$$

where the second inequality in (3.8) holds because $s-\frac{1}{2^{n+1}}<\frac{2\left\lfloor 2^{n-1} s\right\rfloor+\frac{3}{2}}{2^{n}} \leq s+\frac{3}{2^{n+1}}$ for each $n=1,2, \ldots$, and $\lim _{n \rightarrow \infty}\left(s-\frac{1}{2^{n+1}}\right)=\lim _{n \rightarrow \infty}\left(s+\frac{3}{2^{n+1}}\right)=s$; and the equality in (3.8) holds because $\frac{2\left\lfloor 2^{n-1} s\right\rfloor+\frac{3}{2}}{2^{n}} \notin \cup_{k=0}^{2^{n}-1}\left[\frac{2 k}{2^{n}}, \frac{2 k+1}{2^{n}}\right)$ for each $n=1,2, \ldots$ Therefore, equalities (3.7) hold.

We observe that

$$
\liminf _{n \rightarrow \infty, s^{\prime} \rightarrow s} g_{n}\left(s^{\prime}\right)=-\frac{2^{s-1}}{\ln 2} \mathbf{I}\{s \in[0,2]\}, \quad s \in[0,+\infty) .
$$

Indeed, since $g_{n}(s) \geq f_{n}(s)-\frac{2^{s-1}}{\ln 2} \mathbf{I}\{s \in[0,2]\}$ for each $s \in[0,+\infty)$ and $n=1,2, \ldots$, and the function $s \mapsto \liminf _{n \rightarrow \infty, s^{\prime} \rightarrow s} g_{n}\left(s^{\prime}\right)$ is lower semi-continuous, equality (3.9) follows from

$$
\liminf _{n \rightarrow \infty, s^{\prime} \rightarrow s} g_{n}\left(s^{\prime}\right)=\lim _{n \rightarrow \infty} g_{n}\left(\frac{2\left\lfloor 2^{n-1} s\right\rfloor+\frac{1}{2}}{2^{n}}\right)=-\frac{2^{s-1}}{\ln 2}, \quad s \in[0,2),
$$


where the first equality in (3.10) holds because $s-\frac{3}{2^{n+1}}<\frac{2\left\lfloor 2^{n-1} s\right\rfloor+\frac{1}{2}}{2^{n}} \leq s+\frac{1}{2^{n+1}}$ for each $n=1,2, \ldots$, and $\lim _{n \rightarrow \infty}\left(s-\frac{3}{2^{n+1}}\right)=\lim _{n \rightarrow \infty}\left(s+\frac{1}{2^{n+1}}\right)=s$; and the second equality in (3.10) holds because $\frac{2\left\lfloor 2^{n-1} s\right\rfloor+\frac{1}{2}}{2^{n}} \in \cup_{k=0}^{2^{n}-1}\left[\frac{2 k}{2^{n}}, \frac{2 k+1}{2^{n}}\right)$ for $n \geq \max \left\{1,\left\lfloor-\log _{2}(2-s)\right\rfloor\right\}$. Therefore, equality (3.9) holds.

Equality (3.9) implies

$$
\int_{0}^{\infty} \liminf _{n \rightarrow \infty, s^{\prime} \rightarrow s} g_{n}\left(s^{\prime}\right) \mu(d s)=-\int_{0}^{2} \frac{1}{2 \ln 2} d s=-\frac{1}{\ln 2} .
$$

For each $n=1,2, \ldots$,

$$
\int_{0}^{\infty} f_{n}(s) \mu(d s)=-2^{n} \int_{n}^{n+1} 2^{-s} d s=\frac{2^{n}}{\ln 2}\left(2^{-n-1}-2^{-n}\right)=-\frac{1}{2 \ln 2}
$$

and

$$
\int_{0}^{\infty} g_{n}(s) \mu(d s)=\int_{0}^{\infty} f_{n}(s) \mu(d s)-\frac{1}{2 \ln 2} \sum_{k=0}^{2^{n}-1} \frac{1}{2^{n}}=-\frac{1}{\ln 2} .
$$

Inequalities (3.6) hold because, according to (3.11) and (3.13),

$$
-\infty<-\frac{1}{\ln 2}=\int_{\mathbb{S}} \liminf _{n \rightarrow \infty, s^{\prime} \rightarrow s} g_{n}\left(s^{\prime}\right) \mu(d s) \leq \liminf _{n \rightarrow \infty} \int_{\mathbb{S}} g_{n}(s) \mu_{n}(d s)=-\frac{1}{\ln 2} .
$$

However, inequality (2.6) does not hold because, according to (3.12) and (3.7),

$$
-\frac{1}{2 \ln 2}=\liminf _{n \rightarrow \infty} \int_{\mathbb{S}} f_{n}(s) \mu_{n}(d s)<\int_{\mathbb{S}} \liminf _{n \rightarrow \infty, s^{\prime} \rightarrow s} f_{n}\left(s^{\prime}\right) \mu(d s)=0 .
$$

The second inequality in (2.7) does not hold either because, according to (3.13) and (3.7),

$$
-\frac{1}{\ln 2}=\liminf _{n \rightarrow \infty} \int_{\mathbb{S}} g_{n}(s) \mu_{n}(d s)<\int_{\mathbb{S} n \rightarrow \infty, s^{\prime} \rightarrow s} \limsup _{n} g_{n}\left(s^{\prime}\right) \mu(d s)=0 .
$$

Therefore, inequalities (3.6) hold, but inequality (2.6) and the second inequality in (2.7) do not hold.

\section{Examples of applications of Theorem 2.2}

This section provides examples of applications of Theorem 2.2. The usefulness of these applications is that it is typically easier to verify asymptotic u.i. w.r.t. to a sequence of measures than u.i.

\subsection{Uniform Fatou's lemma and uniform Lebesgue's dominated convergence theorem for measures converging in total variation}

The following statements are [16, Theorem 2.1 and Corollary 2.9] with conditions (ii) replacing the conditions that $\left\{f_{n}^{-}\right\}_{n=1,2, \ldots}$ and $\left\{f_{n}\right\}_{n=1,2, \ldots}$ are u.i. w.r.t. $\left\{\mu_{n}\right\}_{n=1,2, \ldots}$ respectively. As explained in [16], inequality (4.1) is stronger than the inequality in Fatou's lemma, and the sufficient condition in Proposition 4.1 can be viewed as the uniform version of Fatou's lemma. Since the convergence in (4.2) is a uniform version of convergence of integrals, the sufficient condition in Proposition 4.2 can be viewed as the uniform version of Lebesgue's dominated convergence theorem. 
Proposition 4.1 (Uniform Fatou's lemma; cp. [16. Theorem 2.1]). Let (S, $\Sigma$ ) be a measurable space, a sequence of measures $\left\{\mu_{n}\right\}_{n=1,2, \ldots}$ from $\mathcal{M}(\mathbb{S})$ converge in total variation to a measure $\mu \in \mathcal{M}(\mathbb{S})$, and $\left\{f, f_{n}\right\}_{n=1,2, \ldots}$ be a sequence of measurable $\overline{\mathbf{R}}$-valued functions on $\mathbb{S}$. Assume that $f \in L^{1}(\mathbb{S} ; \mu)$ and $f_{n} \in L^{1}\left(\mathbb{S} ; \mu_{n}\right)$ for each $n=1,2, \ldots$. Then the inequality

$$
\liminf _{n \rightarrow \infty} \inf _{C \in \Sigma}\left(\int_{C} f_{n}(s) \mu_{n}(d s)-\int_{C} f(s) \mu(d s)\right) \geq 0
$$

takes place if and only if the following two statements hold:

(i) for each $\varepsilon>0$

$$
\mu\left(\left\{s \in \mathbb{S}: f_{n}(s) \leq f(s)-\varepsilon\right\}\right) \rightarrow 0 \text { as } n \rightarrow \infty
$$

(ii) $\left\{f_{n}^{-}\right\}_{n=1,2, \ldots}$ is a.u.i. w.r.t. $\left\{\mu_{n}\right\}_{n=1,2, \ldots}$.

Proof. The theorem follows from [16, Theorem 2.1] and Theorem 2.2 .

We notice that, since $\emptyset \in \Sigma$, the left-hand side of (4.1) is nonpositive. Therefore, inequality (4.1) takes place if and only if it holds in the form of the equality. Since the left hand side of (4.1) is the lower limit of a sequence of nonpositive numbers, the lower limit in (4.1) is the limit.

Proposition 4.2 (Uniform Lebesgue's dominated convergence theorem; cp. [16, Corollary 2.9]). Let $(\mathbb{S}, \Sigma)$ be a measurable space, $\left\{\mu_{n}\right\}_{n=1,2, \ldots}$ be a sequence of measures from $\mathcal{M}(\mathbb{S})$ converging in total variation to a measure $\mu \in \mathcal{M}(\mathbb{S})$, and $\left\{f, f_{n}\right\}_{n=1,2, \ldots}$ be a sequence of measurable $\overline{\mathbf{R}}$-valued functions on $\mathbb{S}$. Assume that $f \in L^{1}(\mathbb{S} ; \mu)$ and $f_{n} \in L^{1}\left(\mathbb{S} ; \mu_{n}\right)$ for each $n=1,2, \ldots$. Then

$$
\lim _{n \rightarrow \infty} \sup _{C \in \Sigma}\left|\int_{C} f_{n}(s) \mu_{n}(d s)-\int_{C} f(s) \mu(d s)\right|=0
$$

if and only if the following two statements hold:

(i) $\left\{f_{n}\right\}_{n=1,2, \ldots}$ converge to $f$ in measure $\mu$;

(ii) $\left\{f_{n}\right\}_{n=1,2, \ldots}$ is a.u.i. w.r.t. $\left\{\mu_{n}\right\}_{n=1,2, \ldots}$.

Proof. The theorem follows from [16, Corollary 2.9] and Theorem 2.2 .

\subsection{On Dunford-Pettis theorem}

As follows from Eberlein-Šmulian theorem, the Dunford-Pettis theorem implies that the sequence $\left\{f_{n}\right\}_{n=1,2, \ldots} \subset L^{1}(\mathbb{S} ; \mu)$ has a weakly convergent subsequence $\left\{f_{n_{k}}\right\}_{k=1,2, \ldots}$ to $f \in L^{1}(\mathbb{S} ; \mu)$ in $L^{1}(\mathbb{S} ; \mu)$ if and only if $\left\{f_{n}\right\}_{n=1,2, \ldots}$ is u.i.; see, for example, Albiac and Kalton [1, Theorem 5.2.9, p. 109], Bogachev [3, Theorem 4.7.18, p. 285], Diestel [8, p. 93], Edwards [10, Theorem 4.21.2, p. 274], Meyer [27, T23, p. 20], Treves [33, Theorem 46.1, p. 471], and Wojtaszczyk 36, Theorem 12, p. 137].

The main result of this subsection has the following formulation.

Proposition 4.3. Let $(\mathbb{S}, \Sigma)$ be a measurable space, $\mu \in \mathcal{M}(\mathbb{S})$, and $\left\{f_{n}\right\}_{n=1,2, \ldots} \subset L^{1}(\mathbb{S} ; \mu)$ be a sequence of measurable $\overline{\mathbf{R}}$-valued functions on $\mathbb{S}$. Then the following statements are equivalent:

(i) there exists $\left\{f_{n_{k}}\right\}_{k=1,2, \ldots} \subset\left\{f_{n}\right\}_{n=1,2, \ldots}$ such that $f_{n_{k}} \rightarrow f$ weakly in $L^{1}(\mathbb{S} ; \mu)$ for some $f \in$ $L^{1}(\mathbb{S} ; \mu)$ 
(ii) there exists $N=0,1, \ldots$ such that $\left\{f_{n+N}\right\}_{n=1,2, \ldots}$ is u.i.;

(iii) $\left\{f_{n}\right\}_{n=1,2, \ldots}$ is a.u.i.

Proof. In view of Eberlein-Šmulian theorem, statements (i) and (ii) are equivalent due to the Dunford-Pettis theorem. The equivalence of statements (ii) and (iii) directly follows from Theorem 2.2.

\subsection{The fundamental theorem for Young measures}

In this subsection we provide an equivalent formulation of the fundamental theorem for Young measures from Ball [2]. Let $n, m=1,2, \ldots, \Omega \subset \mathbf{R}^{n}$ be Lebesgue measurable, $C \subset \mathbf{R}^{m}$ be closed. Let meas denote the Lebesgue measure on $\mathbf{R}^{n}$. Consider Banach spaces $L^{1}(\Omega)$ and $L^{\infty}(\Omega)$ of all integrable and essentially bounded functions on $\Omega$ respectively, endowed with the standard norms.

Proposition 4.4. Let $z^{(j)}: \Omega \mapsto \mathbf{R}^{m}, j=1,2, \ldots$, be a sequence of Lebesgue measurable functions satisfying $z^{(j)}(\cdot) \rightarrow C$ in measure as $j \rightarrow \infty$, that is, for every neighbourhood $U$ of $C$ in $\mathbf{R}^{m}$

$$
\lim _{j \rightarrow \infty} \operatorname{meas}\left\{x \in \Omega: z^{(j)}(x) \notin U\right\}=0 .
$$

Then there exists a subsequence $\left\{z^{\left(j_{k}\right)}\right\}_{k=1,2, \ldots}$ of $\left\{z^{(j)}\right\}_{j=1,2, \ldots}$ and a family $\left(\nu_{x}\right), x \in \Omega$, of positive measures on $\mathbf{R}^{m}$, depending measurably on $x$, such that

(i) $\left\|\nu_{x}\right\|_{M}:=\int_{\mathbf{R}^{m}} d \nu_{x} \leq 1$ for a.e. $x \in \Omega$;

(ii) $\operatorname{supp} \nu_{x} \subset C$ for a.e. $x \in \Omega$; and

(iii) $f\left(z^{\left(j_{k}\right)}\right) \rightarrow\left\langle\nu_{x}, f\right\rangle=\int_{\mathbf{R}^{m}} f(\lambda) d \nu_{x}(\lambda)$ weakly star in $L^{\infty}(\Omega)$ for each continuous function $f: \mathbf{R}^{m} \mapsto \mathbf{R}$ satisfying $\lim _{|\lambda| \rightarrow \infty} f(\lambda)=0$.

Suppose further that $\left\{z^{\left(j_{k}\right)}\right\}_{k=1,2, \ldots}$ satisfies the asymptotic boundedness condition

$$
\lim _{K \rightarrow+\infty} \limsup _{k \rightarrow \infty} \operatorname{meas}\left\{x \in \Omega \cap B_{R}:\left|z^{\left(j_{k}\right)}(x)\right| \geq K\right\}=0,
$$

for every $R>0$, where $B_{R}=B_{R}(\overline{0})$ is a ball of radius $R$ and center $\overline{0}$ in the Euclidean $n$-space $\mathbf{R}^{n}$. Then $\left\|\nu_{x}\right\|_{M}=1$ for a.e. $x \in \Omega$ (that is, $\nu_{x}$ is a probability measure), and given any measurable subset $A$ of $\Omega$

$$
f\left(z^{\left(j_{k}\right)}\right) \rightarrow\left\langle\nu_{x}, f\right\rangle \quad \text { weakly in } L^{1}(A)
$$

for any continuous function $f: \mathbf{R}^{m} \mapsto \mathbf{R}$ such that $\left\{f\left(z^{\left(j_{k}\right)}\right)\right\}_{k=1,2, \ldots}$ is sequentially weakly relatively compact in $L^{1}(A)$.

Remark 4.5. Ball [2, Theorem] is Proposition 4.4 with (4.3) replaced with

$$
\lim _{K \rightarrow+\infty} \sup _{k=1,2, \ldots} \operatorname{meas}\left\{x \in \Omega \cap B_{R}:\left|z^{\left(j_{k}\right)}(x)\right| \geq K\right\}=0 .
$$

Remark 4.6. Condition (4.3) is equivalent to the following one: given any $R>0$ there exists a continuous function $g_{R}:[0,+\infty) \mapsto \mathbf{R}$, with $\lim _{t \rightarrow \infty} g_{R}(t)=+\infty$, such that

$$
\limsup _{k \rightarrow \infty} \int_{\Omega \cap B_{R}} g_{R}\left(\left|z^{\left(j_{k}\right)}(x)\right|\right) d x<\infty
$$

see Ball [2, Remark 1]. Without loss of generality, we can also replace limit superior with limit interior in (4.6) if Condition (4.3) is replaced with the condition in this remark. 
Remark 4.7. If $A$ is bounded in Proposition 4.4, then Proposition 4.3 implies that the condition that $\left\{f\left(z^{\left(j_{k}\right)}\right)\right\}_{k=1,2, \ldots}$ is sequentially weakly relatively compact in $L^{1}(A)$ is satisfied if and only if $\left\{f\left(z^{\left(j_{k}\right)}\right)\right\}_{k=1,2, \ldots}$ is a.u.i.

Proof of Proposition 4.4. All statements follow from Theorem from Ball [2] and Theorem 2.2, being applied to $\mathbb{S}:=\Omega \cap B_{R}$ endowed with the Lebesgue $\sigma$-algebra $\Sigma$ on $\Omega \cap B_{R}$,

$$
\begin{aligned}
f_{k}(s) & :=\left|z^{\left(j_{k}\right)}(s)\right| \mathbf{I}\left\{s \in \mathbb{S}:\left|z^{\left(j_{k}\right)}(s)\right| \geq 1\right\} \\
\mu_{k}(S) & :=\int_{S} \frac{d s}{\left|z^{\left(j_{k}\right)}(s)\right| \mathbf{I}\left\{s \in \mathbb{S}:\left|z^{\left(j_{k}\right)}(s)\right| \geq 1\right\}},
\end{aligned}
$$

for each $S \in \Sigma$ and sufficiently large $k \geq 1$. Indeed, since

$$
\int_{\mathbb{S}}\left|f_{k}(s)\right| \mathbf{I}\left\{s \in \mathbb{S}:\left|f_{k}(s)\right| \geq K\right\} \mu_{k}(d s)=\operatorname{meas}\left\{x \in \Omega \cap B_{R}:\left|z^{\left(j_{k}\right)}(x)\right| \geq K\right\},
$$

for each $K>1$ and sufficiently large $k \geq 1$, we have that Theorem 2.2 implies that (4.3) and (4.5) are equivalent, and, therefore, the statements of Theorem from Ball [2] and Proposition 4.4 are equivalent.

\section{Proofs of Theorems $2.4,2.6$ and Corollary 2.7}

This section contains the proofs of Theorems 2.4, 2.6 and Corollary 2.7.

Proof of Theorem 2.4. Let us fix an arbitrary $K>0$. Then

$$
\begin{aligned}
& \liminf _{n \rightarrow \infty} \int_{\mathbb{S}} f_{n}(s) \mu_{n}(d s) \\
& \geq \liminf _{n \rightarrow \infty} \int_{\mathbb{S}} f_{n}(s) \mathbf{I}\left\{s \in \mathbb{S}: f_{n}(s)>-K\right\} \mu_{n}(d s) \\
& \quad+\liminf _{n \rightarrow \infty} \int_{\mathbb{S}} f_{n}(s) \mathbf{I}\left\{s \in \mathbb{S}: f_{n}(s) \leq-K\right\} \mu_{n}(d s) .
\end{aligned}
$$

The following inequality holds:

$$
\liminf _{n \rightarrow \infty} \int_{\mathbb{S}} f_{n}(s) \mathbf{I}\left\{s \in \mathbb{S}: f_{n}(s)>-K\right\} \mu_{n}(d s) \geq \int_{\mathbb{S}} \liminf _{n \rightarrow \infty, s^{\prime} \rightarrow s} f_{n}\left(s^{\prime}\right) \mu(d s) .
$$

Indeed, if $\mu(\mathbb{S})=0$, then

$$
\liminf _{n \rightarrow \infty} \int_{\mathbb{S}} f_{n}(s) \mathbf{I}\left\{s \in \mathbb{S}: f_{n}(s)>-K\right\} \mu_{n}(d s) \geq-K \lim _{n \rightarrow \infty} \mu_{n}(\mathbb{S})=0=\int_{\mathbb{S}} \liminf _{n \rightarrow \infty, s^{\prime} \rightarrow s} f_{n}\left(s^{\prime}\right) \mu(d s),
$$

where the equalities hold because $\mu_{n}(\mathbb{S}) \rightarrow \mu(\mathbb{S})=0$ as $n \rightarrow \infty$. Otherwise, if $\mu(\mathbb{S})>0$, then [15, Theorem 4.2], applied to $\left\{\tilde{f}_{n}\right\}_{n=1,2, \ldots}:=\left\{f_{n+N}\right\}_{n=1,2, \ldots}, \tilde{g}_{n} \equiv-K, \tilde{\mu}_{n}(C):=\frac{\mu_{n+N}(C)}{\mu_{n+N}(\mathbb{S})}$ and $\tilde{\mu}(C)=\frac{\mu(C)}{\mu(\mathbb{S})}$, for each $n=1,2, \ldots$ and $C \in \mathcal{B}(\mathbb{S})$, where $N=1,2, \ldots$ is sufficiently large, implies

$$
\begin{aligned}
\liminf _{n \rightarrow \infty} & \int_{\mathbb{S}} f_{n}(s) \mathbf{I}\left\{s \in \mathbb{S}: f_{n}(s)>-K\right\} \mu_{n}(d s) \\
& \geq \int_{\mathbb{S}} \liminf _{n \rightarrow \infty, s^{\prime} \rightarrow s} f_{n}\left(s^{\prime}\right) \mathbf{I}\left\{s^{\prime} \in \mathbb{S}: f_{n}\left(s^{\prime}\right)>-K\right\} \mu(d s) .
\end{aligned}
$$


Here we note that $\left\{\tilde{\mu}_{n}\right\}_{n=1,2, \ldots} \subset \mathbf{P}(\mathbb{S})$ converges weakly to $\tilde{\mu} \in \mathbf{P}(\mathbb{S})$. We remark also that

$$
f_{n}(s) \mathbf{I}\left\{s \in \mathbb{S}: f_{n}(s)>-K\right\} \geq f_{n}(s)
$$

for all $s \in \mathbb{S}$ because $K>0$. Thus, (5.2) follows from (5.3) and (5.4).

Inequalities (5.1) and (5.2) imply

$$
\begin{aligned}
\liminf _{n \rightarrow \infty} \int_{\mathbb{S}} f_{n}(s) & \mu_{n}(d s) \geq \int_{\mathbb{S}} \liminf _{n \rightarrow \infty, s^{\prime} \rightarrow s} f_{n}\left(s^{\prime}\right) \mu(d s) \\
+ & \lim _{K \rightarrow+\infty} \liminf _{n \rightarrow \infty} \int_{\mathbb{S}} f_{n}(s) \mathbf{I}\left\{s \in \mathbb{S}: f_{n}(s) \leq-K\right\} \mu_{n}(d s),
\end{aligned}
$$

which is equivalent to (2.6) because $\left\{f_{n}^{-}\right\}_{n=1,2, \ldots}$ is a.u.i. w.r.t. $\left\{\mu_{n}\right\}_{n=1,2, \ldots}$.

Proof of Theorem 2.6. Let Assumption 2.5 hold. According to Theorem 2.2, it is sufficient to prove that $\left\{f_{n}^{-}\right\}_{n=1,2, \ldots}$ is a.u.i. w.r.t. $\left\{\mu_{n}\right\}_{n=1,2, \ldots}$, that is,

$$
\lim _{K \rightarrow+\infty} \liminf _{n \rightarrow \infty} \int_{\mathbb{S}} f_{n}(s) \mathbf{I}\left\{s \in \mathbb{S}: f_{n}(s) \leq-K\right\} \mu_{n}(d s)=0 .
$$

Let us prove (5.5). Indeed, since $f_{n}(s) \geq g_{n}(s)$,

$$
\mathbf{I}\left\{s \in \mathbb{S}: f_{n}(s) \leq-K\right\} \leq \mathbf{I}\left\{s \in \mathbb{S}: g_{n}(s) \leq-K\right\},
$$

for all $n=1,2, \ldots, K>0$, and $s \in \mathbb{S}$. Therefore,

$$
\begin{aligned}
\lim _{K \rightarrow+\infty} & \liminf _{n \rightarrow \infty} \int_{\mathbb{S}} f_{n}(s) \mathbf{I}\left\{s \in \mathbb{S}: f_{n}(s) \leq-K\right\} \mu_{n}(d s) \\
& \geq \lim _{K \rightarrow+\infty} \liminf _{n \rightarrow \infty} \int_{\mathbb{S}} g_{n}(s) \mathbf{I}\left\{s \in \mathbb{S}: g_{n}(s) \leq-K\right\} \mu_{n}(d s) .
\end{aligned}
$$

Inequalities (2.7) imply

$$
\begin{aligned}
\lim _{K \rightarrow+\infty} & \liminf _{n \rightarrow \infty} \int_{\mathbb{S}} g_{n}(s) \mathbf{I}\left\{s \in \mathbb{S}: g_{n}(s) \leq-K\right\} \mu_{n}(d s) \\
& \geq \int_{\mathbb{S}} \limsup _{n \rightarrow \infty, s^{\prime} \rightarrow s} g_{n}\left(s^{\prime}\right) \mu(d s)+\lim _{K \rightarrow+\infty} \liminf _{n \rightarrow \infty} \int_{\mathbb{S}}-g_{n}(s) \mathbf{I}\left\{s \in \mathbb{S}: g_{n}(s)>-K\right\} \mu_{n}(d s) .
\end{aligned}
$$

Since the functions $\left\{g_{n}\right\}_{n=1,2, \ldots}$ are bounded from above by the same constant, Theorem 2.4 applied to the sequence of the functions $\left\{\mathbf{f}_{n}\right\}_{n=1,2, \ldots}$, which are uniformly bounded from below, where $\mathrm{f}_{n}(s):=-g_{n}(s) \mathbf{I}\left\{s \in \mathbb{S}: g_{n}(s)>-K\right\}, s \in \mathbb{S}, n=1,2, \ldots$, implies

$$
\begin{aligned}
\liminf _{n \rightarrow \infty} & \int_{\mathbb{S}}-g_{n}(s) \mathbf{I}\left\{s \in \mathbb{S}: g_{n}(s)>-K\right\} \mu_{n}(d s) \\
& \geq-\int_{\mathbb{S}} \limsup _{n \rightarrow \infty, s^{\prime} \rightarrow s} g_{n}\left(s^{\prime}\right) \mathbf{I}\left\{s^{\prime} \in \mathbb{S}: g_{n}\left(s^{\prime}\right)>-K\right\} \mu(d s)
\end{aligned}
$$

for each $K>0$. If for each $s \in \mathbb{S}$

$$
\limsup _{n \rightarrow \infty, s^{\prime} \rightarrow s} g_{n}\left(s^{\prime}\right) \mathbf{I}\left\{s^{\prime} \in \mathbb{S}: g_{n}\left(s^{\prime}\right)>-K\right\} \downarrow \limsup _{n \rightarrow \infty, s^{\prime} \rightarrow s} g_{n}\left(s^{\prime}\right) \quad \text { as } \quad K \uparrow+\infty,
$$

then (5.6)-(5.8) directly imply (5.5), that is, $\left\{f_{n}^{-}\right\}_{n=1,2, \ldots}$ is a.u.i. w.r.t. $\left\{\mu_{n}\right\}_{n=1,2, \ldots}$. 
Let us prove (5.9). Since for each $s^{\prime} \in \mathbb{S}$ and $n=1,2, \ldots$

$$
g_{n}\left(s^{\prime}\right) \mathbf{I}\left\{s^{\prime} \in \mathbb{S}: g_{n}\left(s^{\prime}\right)>-K\right\} \downarrow g_{n}\left(s^{\prime}\right) \text { as } K \uparrow+\infty,
$$

we have that

$$
\sup _{m \geq n, s^{\prime} \in B_{\delta}(s)} g_{m}\left(s^{\prime}\right) \mathbf{I}\left\{s^{\prime} \in \mathbb{S}: g_{m}\left(s^{\prime}\right)>-K\right\} \downarrow \sup _{m \geq n, s^{\prime} \in B_{\delta}(s)} g_{m}\left(s^{\prime}\right) \quad \text { as } \quad K \uparrow+\infty
$$

for each $n=1,2, \ldots$ and $\delta>0$, where $B_{\delta}(s)$ is the ball in the metric space $\mathbb{S}$ of radius $\delta$ centered at $s$. Therefore,

$$
\inf _{n \geq 1, \delta>0} \sup _{m \geq n, s^{\prime} \in B_{\delta}(s)} g_{m}\left(s^{\prime}\right) \mathbf{I}\left\{s^{\prime} \in \mathbb{S}: g_{m}\left(s^{\prime}\right)>-K\right\} \downarrow \inf _{n \geq 1, \delta>0} \sup _{m \geq n, s^{\prime} \in B_{\delta}(s)} g_{m}\left(s^{\prime}\right) \quad \text { as } \quad K \uparrow+\infty
$$

that is, (5.9) holds for each $s \in \mathbb{S}$. Thus, $\left\{f_{n}^{-}\right\}_{n=1,2, \ldots}$ is u.i. w.r.t. $\left\{\mu_{n}\right\}_{n=1,2, \ldots}$.

Proof of Corollary 2.7. Theorem 2.4, applied to the sequence $\left\{f_{n}-g_{n}\right\}_{n=1,2, \ldots}$ of nonnegative functions, implies

$$
\begin{aligned}
& \int_{\mathbb{S}} \liminf _{n \rightarrow \infty, s^{\prime} \rightarrow s} f_{n}\left(s^{\prime}\right) \mu(d s)-\int_{\mathbb{S}} \limsup _{n \rightarrow \infty, s^{\prime} \rightarrow s} g_{n}\left(s^{\prime}\right) \mu(d s) \\
& \leq \int_{\mathbb{S}} \liminf _{n \rightarrow \infty, s^{\prime} \rightarrow s}\left(f_{n}\left(s^{\prime}\right)-g_{n}\left(s^{\prime}\right)\right) \mu(d s) \leq \liminf _{n \rightarrow \infty} \int_{\mathbb{S}}\left(f_{n}(s)-g_{n}(s)\right) \mu_{n}(d s) \\
& \leq \liminf _{n \rightarrow \infty} \int_{\mathbb{S}} f_{n}(s) \mu_{n}(d s)-\liminf _{n \rightarrow \infty} \int_{\mathbb{S}} g_{n}(s) \mu_{n}(d s),
\end{aligned}
$$

where the first and the third inequalities follow from the basic properties of infimums and supremums. Inequality (2.6) follows from (5.10) and Assumption 2.5.

Acknowledgements. The research of the first and the third author was supported by NSF Grant CMMI-1636193. The authors thank G. M. Shevchenko for bringing their attention to M. V. Kartashov's book [25].

\section{REFERENCES}

[1] F. Albiac and N. J. Kalton, Topics in Banach Space Theory, 2nd ed., Springer International Publishing, Switzerland, 2016.

[2] J. M. BALL, A version of the fundamental theorem for Young measures, in M. Rascle, D. Serre, and M. Slemrod (eds.), Proceedings of Conference on "Partial Differential Equations and Continuum Models of Phase Transitions", pages 3-16, Lecture Notes in Physics, No. 359, Springer, Berlin, 1989.

[3] V. I. Bogachev, Measure Theory, Volume I, Springer-Verlag, Berlin, 2007.

[4] V. S. Borkar, Convex Analytic Methods in Markov Decision Processes, in E.A. Feinberg and A. Shwartz (eds,), Handbook of Markov Decision Processes, pages 347-575, Kluwer, Boston, 2002 
[5] J. P. Chen And B. E. UGurcan, Entropic repulsion of Gaussian free field on highdimensional Sierpinski carpet graphs, Stoch. Proc. Appl., 125 (2015), pp. 4632-4673.

[6] S. Chu And Y. Zhang, Markov decision processes with iterated coherent risk measures, Int. J. Control, 87 (2014), pp. 2286-2293.

[7] K. DȩBicki, S. Engelke, And E. Hashorva, Generalized Pickands constants and stationary max-stable processes, Extremes, 20 (2017), pp. 493-517.

[8] J. Diestel, Sequences and Series in Banach Spaces, Springer-Verlag, New York, 1984.

[9] F. Dufour and T. Prieto-Rumeau, Conditions for solvability of the linear programming formulation for constrained discounted Markov decision processes, Appl. Math. Optim., 74 (2016), pp. 27-51.

[10] R. E. Edwards, Functional Analysis: Theory and Applications, Rinehart and Winston, New York, 1965.

[11] E. A. Feinberg, Optimality Conditions for Inventory Control, in A. Gupta and A. Capponi (eds.), Optimization Challenges in Complex, Networked, and Risky Systems, Tutorials in Operations Research, pages 14-44, INFORMS, Cantonsville, MD, 2016.

[12] E. A. Feinberg And J. HuAng, Reduction of total-cost and average-cost MDPs with weakly continuous transition probabilities to discounted MDPs, Oper. Res. Lett., 46 (2018), pp. 179 184.

[13] E. A. Feinberg, A. Jaśkiewicz, And A. S. Nowak, Constrained discounted Markov decision processes with Borel state spaces, 2019, https://arxiv.org/pdf/1806.00190.pdf .

[14] E. A. Feinberg, P. O. Kasyanov, and N. V. Zadoianchuk, Average-cost Markov decision processes with weakly continuous transition probabilities, Math. Oper. Res., 37 (2012), pp. 591607.

[15] E. A. Feinberg, P. O. Kasyanov, and N. V. Zadoianchuk, Fatou's lemma for weakly converging probabilities, Theory Probab. Appl., 58 (2013), pp. 812-818.

[16] E. A. Feinberg, P. O. Kasyanov, and M. Z. Zgurovsky, Uniform Fatou's lemma, J. Math. Anal. Appl., 444 (2016), pp. 550-567.

[17] E. A. Feinberg, P. O. Kasyanov, and M. Z. Zgurovsky, Partially observable total-cost Markov decision processes with weakly continuous transition probabilities Math. Oper. Res., 41 (2016), pp. 656-681.

[18] E. A. Feinberg And M. E. Lewis, On the convergence of optimal actions for Markov decision processes and the optimality of $(s, S)$ policies for inventory control, Naval Res. Logist., 65 (2018), pp. 619-637.

[19] N. García Trillos And D. Sanz-Alonso, Continuum limits of posteriors in graph Bayesian inverse problems, SIAM J. Math. Anal., 50 (2018), pp. 4020-4040.

[20] F. Gonçalves, A central limit theorem for operators, J. Funct. Anal., 271 (2016), pp. 15851603. 
[21] E. Hashorva, Representations of max-stable processes via exponential tilting, Stochastic Process. Appl., 128(2018), pp. 2952-2978.

[22] A. JaŚKIEWICZ AND A. S. NowaK, Zero-sum ergodic stochastic games with Feller transition probabilities, SIAM J. Control Optim., 45 (2006), pp. 773-789.

[23] A. JaśKIEwicz And A. S. NowaK, Stochastic games with unbounded payoffs: Applications to robust control in economics, Dyn. Games. Appl., 1 (2011), pp. 253-279.

[24] T. Kaminigashi, A generalization of Fatous lemma for extended real-valued functions on $\sigma$ finite measure spaces: with an application to infinite-horizon optimization in discrete time, J. Inequal Appl., 2017(1):24, doi:10.1186/s13660-016-1288-5.

[25] M. V. Kartashov, Probability, Processes, Statistics, Kyiv University Press, Kyiv, 2008 (in Ukrainian).

[26] D. Landriault, B. Li, And H. Zhang, A unified approach for drawdown (drawup) of timehomogeneous Markov processes, J. Appl. Probab., 54 (2017), pp. 603-626.

[27] P. A. Meyer, Probability and Potential, Blaisdell Pub. Co., Waltham, MA, 1966.

[28] X. Ren, J. Wu, K. H. Johansson, and G. Shi, Infinite Horizon Optimal Transmission Power Control for Remote State Estimation Over Fading Channels, IEEE Trans. Autom. Control, 63 (2018), pp. 85-100.

[29] R. Serfozo, Convergence of Lebesgue integrals with varying measures, Sankhyā Ser. A., 44 (1982), pp. 380-402.

[30] A. N. Shiryaev, Probability, 2nd ed., Springer-Verlag, New York, 1996.

[31] M. Thorpe And A. M. Johansen, Pointwise convergence in probability of general smoothing splines, Ann. Inst. Stat. Math., 70 (2018), pp. 717-744.

[32] M. Thorpe, F. Theil, A. M. Johansen, And N. Cade, Convergence of the k-means minimization problem using $\Gamma$-convergence, SIAM J. Appl. Math., 75 (2015), pp. 2444-2474.

[33] F. Treves, Topological Vector Spaces, Distributions and Kernels, Academic Press, New York, 1967.

[34] A. D. van der VaArt, Asymptotic Statistics, Cambridge University Press, Cambridge, 1998.

[35] Ó. Vega-AmayA, Solutions of the average cost optimality equation for Markov decision processes with weakly continuous kernel: The fixed-point approach revisited, J. Math. Anal. Appl., 426 (2015), pp. 978-985.

[36] P. Wojtaszczyk, Banach Spaces for Analysts, Cambridge University Press, Cambridge, 1991.

[37] H. XU, Y. LiU, AND H. Sun, Distributionally robust optimization with matrix moment constraints: Lagrange duality and cutting plane methods, Math. Prog. Ser. A, 169 (2018), pp. 489-529. 


\section{Appendix: Proof of Theorem 2.2}

This appendix contains the proof of Theorem 2.2. This proof is close to the proof of the similar result in Kartashov [25, p. 180] for the case of a single probability measure. The main reason for providing this appendix is that reference [25] may not be available for the majority of the readers. The authors of this note have not been aware about [25] for long time. After the first version of this note, which contained a direct proof of Theorem 2.2, was posted in arxiv.org, Professor G.M. Shevchenko informed the authors about the book by M.V. Kartashov [25].

Lemma A.1 (Kartashov [25, p. 134]). Consider a sequence of real-valued functions $\varepsilon_{n}(K)$, where $K>0$, such that

(a) $\varepsilon_{n}(K) \downarrow 0$ as $K \rightarrow+\infty$ for each $n=1,2, \ldots$; and

(b) $\lim _{K \rightarrow+\infty} \lim \sup _{n \rightarrow \infty} \varepsilon_{n}(K)=0$.

Then $(\mathrm{c}) \lim _{K \rightarrow+\infty} \sup _{n=1,2, \ldots} \varepsilon_{n}(K)=0$.

Proof. On the contrary assume that (c) does not hold. In this case the limit in (c) is equal to $\delta$ for some $\delta>0$. Observe that $\sup _{n=1,2, \ldots} \varepsilon_{n}(K) \geq \delta$ for all $K>0$ since this function does not increase in $K$. For each $m=1,2, \ldots$ there is a natural number $n_{m}$ such that $\varepsilon_{n_{m}}(m) \geq \delta / 2$. If the sequence $\left\{n_{m}\right\}_{m=1,2, \ldots}$ is bounded, then it is possible to choose $n_{m}=k$ for some $k \in\{1,2, \ldots\}$ and for an infinite subset of integer numbers $m$. Therefore, $\varepsilon_{k}(m) \geq \delta / 2$ for these numbers, which contradicts assumption (a). For $m>K$, as follows from monotonicity in (a), $\varepsilon_{n}(K) \geq \varepsilon_{n}(m)$, which implies

$$
\limsup _{n \rightarrow \infty} \varepsilon_{n}(K) \geq \limsup _{m \rightarrow \infty} \varepsilon_{n_{m}}(K) \geq \limsup _{m \rightarrow \infty} \varepsilon_{n_{m}}(m) \geq \delta / 2>0,
$$

where $K>0$ is an arbitrary real number, and (A.1) contradicts condition (b).

Proof of Theorem 2.2. The uniform integrability w.r.t. $\left\{\mu_{n+N}\right\}_{n=1,2, \ldots}$ of the sequence $\left\{f_{n+N}\right\}_{n=1,2, \ldots}$ for some $N=0,1, \ldots$ implies the asymptotic uniform integrability w.r.t. $\left\{\mu_{n}\right\}_{n=1,2, \ldots}$ of the sequence $\left\{f_{n}\right\}_{n=1,2, \ldots}$.

Vice versa, let $\left\{f_{n}\right\}_{n=1,2, \ldots}$ is a.u.i. w.r.t. $\left\{\mu_{n}\right\}_{n=1,2, \ldots}$. Then there exists $N=0,1, \ldots$ such that $f_{n} \in L^{1}\left(\mathbb{S} ; \mu_{n}\right)$ for each $n=N+1, N+2, \ldots$. Indeed, if there exists a subsequence $\left\{f_{n_{k}}\right\}_{k=1,2, \ldots}$ of $\left\{f_{n}\right\}_{n=1,2, \ldots}$ such that $\int_{\mathbb{S}}\left|f_{n_{k}}(s)\right| \mu_{n_{k}}(d s)=\infty$, then $\int_{\mathbb{S}}\left|f_{n_{k}}(s)\right| \mathbf{I}\left\{s \in \mathbb{S}:\left|f_{n_{k}}\right| \geq K\right\} \mu_{n_{k}}(d s)=\infty$ for each $K>0$ and $k=1,2, \ldots$. Therefore,

$$
\lim _{K \rightarrow+\infty} \limsup _{n \rightarrow \infty} \int_{\mathbb{S}}\left|f_{n}(s)\right| \mathbf{I}\left\{s \in \mathbb{S}:\left|f_{n}(s)\right| \geq K\right\} \mu_{n}(d s)=\infty
$$

which contradicts the assumption that $\left\{f_{n}\right\}_{n=1,2, \ldots}$ is a.u.i. w.r.t. $\left\{\mu_{n}\right\}_{n=1,2, \ldots}$.

Consider $\varepsilon_{n}(K):=\int_{\mathbb{S}}\left|f_{n+N}(s)\right| \mathbf{I}\left\{s \in \mathbb{S}:\left|f_{n+N}(s)\right| \geq K\right\} \mu_{n+N}(d s), n=1,2, \ldots$. Since $f_{n+N} \in$ $L^{1}\left(\mathbb{S} ; \mu_{n+N}\right)$ for each $n=1,2, \ldots$, condition (a) in Lemma A.1 holds. Condition (b) in Lemma A.1 holds because $\left\{f_{n}\right\}_{n=1,2, \ldots}$ is a.u.i. w.r.t. $\left\{\mu_{n}\right\}_{n=1,2, \ldots}$. Then Lemma A.1 implies the validity of (2.1) for $\left\{f_{n+N}\right\}_{n=1,2, \ldots}$ w.r.t. $\left\{\mu_{n+N}\right\}_{n=1,2, \ldots}$. 